\title{
The Nucleolus of the Bankruptcy Problem by Hydraulic Rationing
}

\author{
Tamás Fleiner* Balázs Sziklai ${ }^{\dagger}$
}

September 2, 2014

\begin{abstract}
In this note, we give a straightforward and elementary proof of a theorem by Aumann and Maschler stating that in the well-known bankruptcy problem, the so called CG-consistent solution described by the Talmud represents the nucleolus of the corresponding coalitional game. The proof nicely fits into the hydraulic rationing framework proposed by Kaminski. We point out further interesting properties in connection with this framework.
\end{abstract}

\section{Introduction}

The bankruptcy problem is one of the oldest in the history of economics. In its simplest form, we have a bankrupt firm and creditors that wish to collect their claims. The total demand exceeds the firm's liquidation value. A natural question is how to divide this value among the claimants. Depending on the notion of fairness, one can impose many rules for such a division. For an an excellent survey on this matter see [10].

One of the oldest concepts can be found in the Talmud [8]. The proposed solution is puzzling at first nevertheless it exhibits many nice properties. It is a mixture of the concepts of constrained equal losses and constrained equal awards. The underlying rationing was not clear until Aumann and Maschler solved the riddle in 1985 [2]. They gave an elementary proof showing that each bankruptcy problem has a so called CG-consistent solution. Moreover, this coincides with the examples in the Talmud. They also showed

*Budapest University of Technology and Economics, Department of Computer Science and Information Theory, H-1117 Budapest, Magyar tudósok körútja 2. Email: fleiner@cs.bme.hu . Research was supported by the OTKA K 69027 project and the MTA-ELTE Egerváry Research Group.

${ }^{\dagger}$ Institute of Economics, HAS, H-1112, Budapest, Budaörsi út 45. Email: sziklai@econ.core.hu . The author thanks the funding of the Hungarian Academy of Sciences under its Momentum Programme (LD-004/2010). 
that the CG-consistent solution is the nucleolus of the corresponding bankruptcy game. This is an interesting result that comes with a less elementary proof.

In 2000, Kaminski introduced a fascinating new concept to represent the bankruptcy game and other similar problems [6]. In his hydraulic rationing, he proposed a physical device wherein vessels correspond to claims and water corresponds to the firm's liquidation value.

Here we show an elementary proof of the nucleolus property using Kaminski's idea. In many games especially in the case of bankruptcy games the negative excess of a coalition can be more descriptive than simple excess. Translating the notion of negative excess into the language of hydraulics is one of the key ideas of the proof. Our approach seems to be more direct than that of Benoît published in 1997 [3].

\section{The Model}

In this section, we describe the basic notions of the bankruptcy problem. Readers familiar with these concepts might want to skip this part and jump to the next section. For more on the models of fair allocation see [11].

Let $N=\{1,2, \ldots, n\}$ be a set of agents. The bankruptcy problem is defined as a pair $(c, E)$ where $E \in \mathbb{R}_{+}$is the firm's liquidation value (or estate/endowment) and $c \in \mathbb{R}_{+}^{n}$ is the collection of claims with $\sum_{i}^{n} c_{i}>E$. Let $\mathbb{B}$ denote the class of such problems. A solution of a bankruptcy problem is a vector $\mathbf{x} \in \mathbb{R}_{+}^{n}$ such that $\sum_{i}^{n} x_{i}=E$. For convenience, we introduce $x(S)=\sum_{i \in S} x_{i}$ and $c(S)=\sum_{i \in S} c_{i}$ for any $S \subseteq N$. Hence $x \in \mathbb{R}_{+}^{n}$ is a solution if $x(N)=E$. A rule $r: \mathbb{B} \rightarrow \mathbb{R}^{n}$ is a mapping that assigns a unique solution to each bankruptcy problem. The dual of a rule $r$ (denoted by $r^{*}$ ) assigns awards in the same way as $r$ assigns losses, namely $r^{*}(c, E)=c-r(c, c(N)-E)$. A self-dual rule is one with $r^{*}=r$, such rule treats losses and awards the same way.

We formalize four rules that we will need later. The reader can find several characterizations of these rules in [4] and [7].

The constrained equal-awards (CEA) rule assigns equal awards to each agent subject to no one receiving more than his claim. The dual of this rule is the constrained equallosses (CEL) rule. In this case losses are distributed as equally as possible subject to no one receives a negative amount. Formally:

Constrained equal-awards: For all $(c, E) \in \mathbb{B}$ and $i \in N, C E A_{i}(c, E)=\min \left(c_{i}, \lambda\right)$ where $\lambda$ solves $\sum \min \left(c_{i}, \lambda\right)=E$.

Constrained equal-losses: For all $(c, E) \in \mathbb{B}$ and $i \in N, C E L_{i}(c, E)=\min \left(0, c_{i}-\right.$ $\lambda)$ where $\lambda$ solves $\sum \min \left(0, c_{i}-\lambda\right)=E$.

Another well-known rule is the random arrival rule. Suppose the claims arrive sequentially and they are fully compensated until the money runs out. The random arrival 
rule computes award vectors for every possible ordering of claims and then takes the average. Hence it produces the same award vector as the Shapley-value applied to the corresponding bankruptcy game[8].

The Contested Garment Principle is a division formula that can be derived from the Talmud. There are two claimants and a divisible piece of good. According the principle, each claimant should give the part of the good that he does not contest to the other claimant. Then the rest is split up equally.

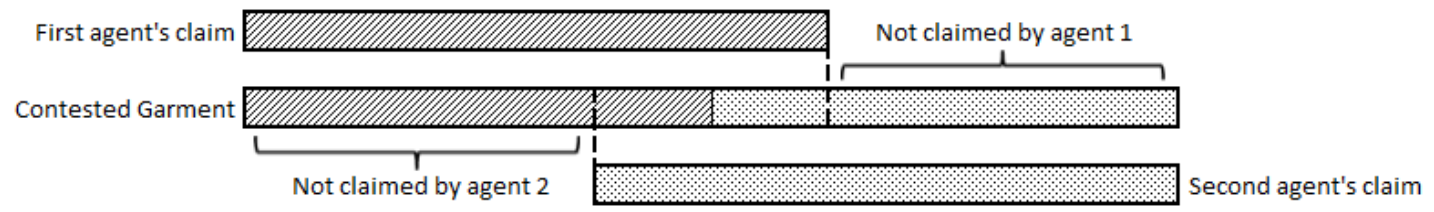

Figure 1: The Contested Garment Principle

Note that the CG-principle does not distinguish between claims that exceed the size of the estate. Claims that are larger than the estate are treated as if they were 'only' claiming the whole and not more.

Definition 1. Let $r(c, E)$ be a division rule defined on each $(c, E) \in \mathbb{B}$. We say that $r$ is pairwise consistent if for all $N^{\prime} \subset N$, where $\left|N^{\prime}\right|=2$ if $x \equiv r(c, E)$, then $x_{N^{\prime}}=$ $r\left(c_{N^{\prime}}, \sum_{N^{\prime}} x_{i}\right)$

The stronger version, consistency ${ }^{1}$ is obtained by dropping the restriction $\left|N^{\prime}\right|=2$. For more on this topic see [5].

We refer to the pairwise consistent rule that divides the endowment between any two agent by the Contested Garment Principle as the $C G$-consistent solution.

Definition 2. Let $(c, E)$ be a bankruptcy problem. A solution is called CG-consistent, if for all $i \neq j$ the division of $x(i)+x(j)$ prescribed by the contested garment principle for claims $c_{i}, c_{j}$ is $(x(i), x(j))$.

The CG-consistent solution (or Talmud rule) can be written formally as

$$
T_{i}(c, E)= \begin{cases}\min \left\{c_{i} / 2, \lambda\right\} & \text { if } E \leq \frac{1}{2} c(N), \\ \max \left\{c_{i} / 2, c_{i}-\mu\right\} & \text { if } E>\frac{1}{2} c(N),\end{cases}
$$

where $\lambda$ and $\mu$ are chosen so that $\sum_{i \in N} T_{i}(c, E)=E$.

Observe that the Talmud rule is the combination of the constrained equal awards and the constrained equal losses rules.

\footnotetext{
${ }^{1}$ In the paper of Aumann and Maschler this property is called self-consistency [2].
} 


\section{$3 \quad$ Hydraulic rationing}

Proof techniques that use the principles of mechanics were very common in the ancient times $^{2}$. The increasing number of examples in the literature shows that these are no less useful today. Just to mention some well-known instances: the shortest path in a directed graph can be found using a system of strings and knots, congestion games can be represented by electric circuits, and - as in our case - rationing problems can be modeled using hydraulic systems.

To prove the Aumann-Maschler result on the nucleolus, we will employ Kaminski's idea and construct a specific hydraulic. In this hydraulic, every claim is represented by a vessel while the firm's liquidation value corresponds to the amount of water we pour into this system. Our vessels have a peculiar hourglass-shape with the following characteristics:

- Each vessel has an upper and lower tank of a shape of a cylinder.

- The upper and lower tanks have the same volume and they are connected with a capillary.

- The capillaries have negligible volume.

- The cylinders have a circular base with area of 1 .

- The volume of vessel $i$ is equal to the size of agent $i$ 's claim.

- Finally each vessel has the same height denoted by $h$. We may assume $h=c_{\max }$ where $c_{\max }$ denotes the largest claim.

Note that the last condition implies that the vessel with the largest volume has no capillary part. We say that a hydraulic is talmudic if it incorporates the above characteristics.

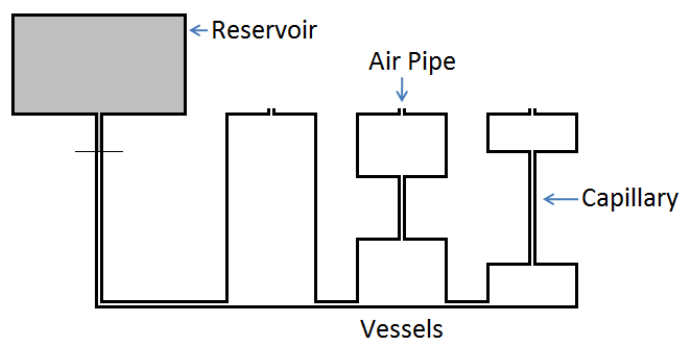

Figure 2: A connected talmudic hydraulic

\footnotetext{
${ }^{2}$ Archimedes wrote to Eratosthenes: "I thought fit to write out for you and explain in detail... a certain method, by which it will be possible for you... to investigate some of the problems in mathematics by means of mechanics. This procedure is, I am persuaded, no less useful even for the proof of the theorems themselves..."
} 
It is not included in the above list but for a proper representation every vessel has to have also a capillary on the top tank where the air can leave the vessel. During the proof, we will need two types of vessel systems (or hydraulics). In a connected hydraulic, vessels are connected with capillaries at the bottom. This way, if we pour water into any of the vessels each vessel starts to fill up. In case of a disconnected hydraulic, vessels are not connected by capillaries. Thus different vessels can have different 'water levels' depending on how much water we pour into them.

We say that a hydraulic $\mathcal{H}$ corresponds to a bankruptcy problem $(\mathrm{c}, \mathrm{E})$ if the following conditions hold

- $\mathcal{H}$ has $n$ vessels

- the volume of the $i$ th vessel is equal to the size of the claim of agent $i$

- there is $E$ amount of water distributed among the vessels.

A hydraulic that corresponds to a bankruptcy problem always implicitly defines an allocation rule. The nature of the rule depends on the shape of the vessels.

Here we show the representation of the rules we have already mentioned.
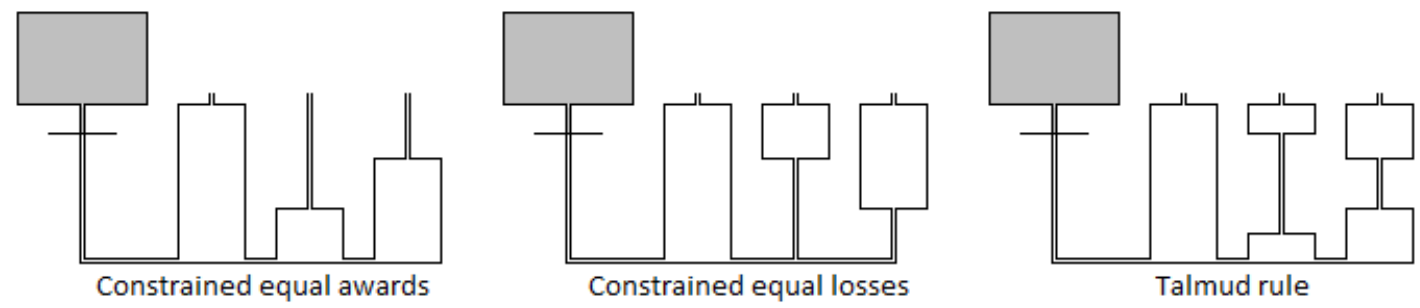

Figure 3: The representation of CEA, CEL and Talmud rules

Observation 3. (Kaminski) Let $(c, E)$ be a two-person bankruptcy problem and let $\mathcal{H}$ be the corresponding connected talmudic hydraulic. The solution $x$ defined by the common water level $z$ in $\mathcal{H}$ is $C G$-consistent.

Indeed, it is not hard to check that the common water level $z$ induces the same allocations as the CG-principle. For a detailed explanation the reader is referred to [1] or [6].

Corollary 4. Each bankruptcy problem has a unique CG-consistent solution.

To further illustrate the robustness of Kaminski's method, we observe an interesting property of consistent solutions.

Observation 5. A rule is consistent if and only if it corresponds to a connected hydraulic in which a shape of a vessel depends only on the respective claim size. 
Consistency of a rule means that a connected hydraulic corresponds to the same rule after removing some of its vessels. Note that the Random arrival rule is an example of a rule that is non consistent. This is so because if a claim disappears then the shape of the remaining vessels has to change.

Another remarkable feature of the hydraulic approach is that it characterizes selfduality in a succinct way.

Observation 6. The Talmud rule is a self-dual one.

Let $\mathcal{H}$ be a connected talmudic hydraulic with $E$ amount of water in it. We have to show that the corresponding rule $T$ is a self-dual rule, or formally, that

$$
T^{*}(c, E)=c-T(c, c(N)-E) .
$$

We can translate this into the language of hydraulics. Let $x=\left(x_{1}, x_{2}, \ldots, x_{n}\right)$ be the solution induced by the common water level. Now consider a copy of $\mathcal{H}$ which is fully filled with water. Let $y=\left(y_{1}, y_{2}, \ldots, y_{n}\right)$ be the distribution of air when we let out $E$ amount of water from the fully filled hydraulic. As the figure shows the distributions are the same.

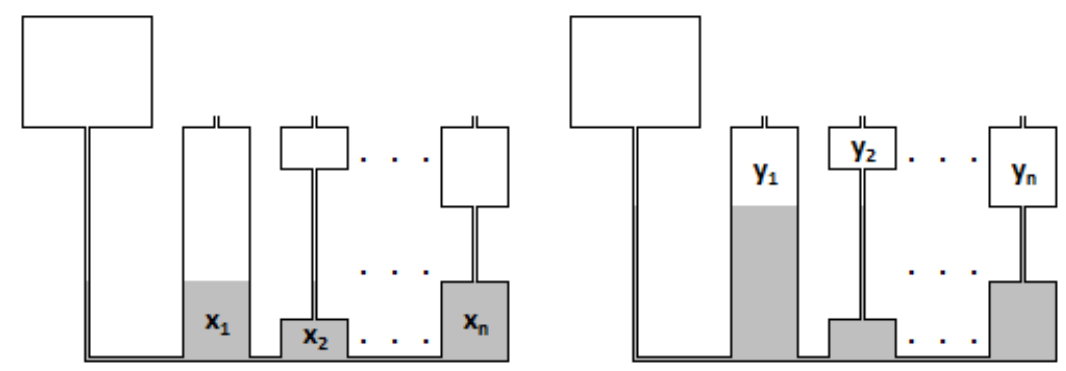

Figure 4: The hydraulic $\mathcal{H}$ with $E$ and $c(N)-E$ amount of water in it.

Actually more is true. Easy to conclude the following fact.

Observation 7. A rule is self-dual if and only if it corresponds to some horizontally symmetric connected hydraulic.

\section{The Nucleolus of the Bankruptcy Game}

Bankruptcy problems can be modeled as coalitional games. Let us recall some basic notions of cooperative games. A cooperative game in characteristic function form is an ordered pair $(N, v)$ consisting of the player set $N=\{1,2, \ldots, n\}$ and a characteristic function $v: 2^{N} \rightarrow \mathbb{R}$ with $v(\emptyset)=0$. Furthermore $x \in \mathbb{R}^{n}$ is called an allocation if it is 
efficient i.e. if $x(N)=v(N)$. We say that an allocation $x$ is an imputation or individually rational if $x(i) \geq v(i)$ for all $i \in N$. The set of imputation is denoted by $I(N, v)$.

$$
I(N, v)=\left\{x \in \mathbb{R}^{n} \mid x(N)=v(N), x(i) \geq v(i) \text { for all } i \in N\right\} .
$$

Given an allocation $x \in \mathbb{R}^{n}$, we define the profit (or negative excess) of a coalition $S$ as

$$
p(S, x):=x(S)-v(S) .
$$

Let $\theta(x) \in \mathbb{R}^{2^{n}}$ be the profit vector that contain the $2^{n}$ profit values in a non-decreasing order.

We say that a vector $x \in \mathbb{R}^{m}$ lexicographically precedes $y \in \mathbb{R}^{m}$ (denoted by $x \preceq y$ ) if either $x=y$ or there exists a number $1 \leq j<m$ such that $x_{i}=y_{i}$ if $i \leq j$ and $x_{j+1}<y_{j+1}$.

Definition 8. The nucleolus is the vector of allocations of a game $x \in \mathbb{R}_{+}^{n}$ that lexicographically maximizes $\theta(x)$ over $I(N, v)^{3}$. In other words,

$$
N(v)=\{x \in I(N, v) \mid \theta(y) \preceq \theta(x) \text { for all } y \in I(N, v)\}
$$

Let $N=\{1,2, \ldots, n\}$ be a set of agents and $(c, E) \in \mathbb{B}$ a bankruptcy problem. For any $S \in 2^{N}$ the characteristic function of the related bankruptcy game is

$$
v_{(c, E)}(S)=\max (E-c(N \backslash S), 0)
$$

The characteristic function represents the worth of each coalition $S$. By definition, it is the value that is left from the firm's liquidation value $E=v(N)$ after the claim of each agent of the complement coalition $N \backslash S$ has been satisfied. This is the value that coalition $S$ can achieve without any effort. Recall that the profit of coalition $S$ is $p(x, S)=x(S)-v_{(c, E)}(S)$. If a coalition $S$ has nothing after all other claimants outside the coalition have been paid off then its profit will be $x(S)$. Otherwise the gain of $S$ should be decreased by $v_{(c, E)}(S)$ since $S$ would get $v_{(c, E)}(S)$ anyway.

We need two small observations. Let $\mathcal{H}$ be a disconnected hydraulic. Now $x(S)$ is the amount of water that is distributed among the vessels that belong to coalition $S$. The next lemma shows that the profit of coalition $S$ is the minimum of the following two amounts: the water contained in $S$ and the air contained in $N \backslash S$.

Lemma 9. Let $v_{(c, E)}$ be a bankruptcy game on player set $N$ and $x$ an imputation. The profit of $S \subseteq N$ can be written as

$$
p(x, S)=\min (x(S), c(N \backslash S)-x(N \backslash S)) .
$$

\footnotetext{
${ }^{3}$ It is well-known that the nucleolus of any cooperative game is unique. This is because if two different allocations $x$ and $y$ have the same profit vector then $\theta(x)=\theta(y) \prec \theta\left(\frac{1}{2}(x+y)\right)[9]$.
} 
Proof. We apply the definition:

$$
\begin{gathered}
p(x, S)=x(S)-v_{(c, E)}(S)=x(S)-\max (0, E-c(N \backslash S))=x(S)+\min (0,-E+c(N \backslash S))= \\
=\min (x(S), x(S)-E+c(N \backslash S))=\min (x(S), x(S)-x(N)+c(N \backslash S))= \\
=\min (x(S), c(N \backslash S)-x(N \backslash S))
\end{gathered}
$$

Our second observation is that the CG-consistent solution coincides with the nucleolus for two person bankruptcy games. We prove this with the following lemma.

Lemma 10. If $(c, E)$ is a two-person bankruptcy game with $N=\{1,2\}$ and $x$ is a solution of it then $p(\{1\}, x)+p(\{2\}, x)=E-\left(v_{(c, E)}(\{1\})+v_{(c, E)}(\{2\})\right)$. This means that the sum of the profits of the two players is exactly the doubly claimed amount (i.e. the part of estate that is not conceded by any of the agents).

Proof. By definition, $p(\{1\}, x)+p(\{2\}, x)=x(\{1\})-v_{(c, E)}(\{1\})+x(\{2\})-v_{(c, E)}(\{2\})=$ $x(\{1\})+x(\{2\})-\left(v_{(c, E)}(\{1\})+v_{(c, E)}(\{2\})\right)=E-\left(v_{(c, E)}(\{1\})+v_{(c, E)}(\{2\})\right)$. As we have seen, $v_{(c, E)}(\{i\})$ is that part of the estate that the other player does not claim, the right hand side is the exactly the doubly clamied part.

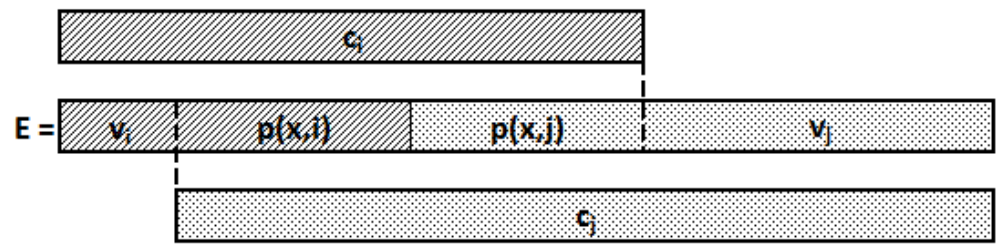

Figure 5: The doubly contested part is divided equally by the CG rule.

Lemma 11. If $(c, E)$ is a two-person bankruptcy game then its $C G$-consistent solution is the nucleolus of the corresponding coalitional game.

Proof. As $p(x, \emptyset)=p(x, N)=0$ for any solution $x$ of the game, the nonzero coordinates of $\theta(x)$ are the profits of the single players. As the sum of these profits are constant by Lemma 10, the profit vector is lexicographically increasing if we decrease the share of the player with the greater profit and increase the share of the player with the smaller profit. This means that the nucleolus is that solution where the profits of the two players are equal, that is, where the doubly claimed part is halved. This is exactly the CG-consistent solution.

Now we are ready to formulate the elementary proof we promised.

Theorem 12 (Aumann-Maschler [2]). The CG-consistent solution of a bankruptcy problem is the nucleolus of the corresponding coalitional game. 
Proof. Let $t$ denote the CG-consistent solution of bankruptcy game $(c, E)$ and assume $x \neq t$ for some solution $x$ of the game. We shall show that there is another solution $x^{\prime}$ lexicographically greater than $x$ such that $x^{\prime}$ and $t$ agrees in more coordinates than $x$ and t. Clearly, this immediately implies Theorem 12.

As $x$ and $t$ are different solutions, there are players $i$ and $j$ such that $x_{i}>t_{i}$ and $x_{j}<t_{j}$. Construct solution $x^{\prime}$ from $x$ such that we start to decrease $i$ 's share and increase $j$ 's share until we reach $x_{i}^{\prime}=t_{i}$ or $x_{j}^{\prime}=t_{j}$. (In other words, if we start from disconnected hydraulic representing $x$ then we let some water from vessel $i$ to vessel $j$ until one of the water levels reaches the water level of $t$ that is strictly between the two original levels.) Clearly, $x^{\prime}$ agrees with $t$ on more coordinates than $x$ did.

Observe that if $p(x, S) \neq p\left(x^{\prime}, S\right)$ then either $i \in S \not \supset j$ and $p\left(x^{\prime}, S\right)<p(x, S)$ or $i \notin S \ni j$ and $p\left(x^{\prime}, S\right)>p(x, S)$. Let $S_{j, \bar{i}}$ denote the set of coalitions that contain $j$ but not $i$. Therefore, to show that $\theta\left(x^{\prime}\right) \succ \theta(x)$, it is enough to prove that any decreased profit is at least as great as the smallest increased profit, that is

$$
\min _{S \in S_{i, \bar{j}}} p\left(x^{\prime}, S\right) \geq \min _{S \in S_{j, \bar{i}}} p\left(x^{\prime}, S\right) .
$$

If we think about the disconnected hydraulic that corresponds to $x^{\prime}$ then the profit is either the amount of water in $S$, namely $x(S)$ or the amount of air in the complement coalition $N \backslash S$, namely $c(N \backslash S)-x(N \backslash S)$. By Lemma 9 , this is the minimum of these two amounts. Observe that the smaller the coalition $S$ the smaller the value $x(S)$. Moreover, the bigger the coalition $S$ the smaller the value $c(N \backslash S)-x(N \backslash S)$. Therefore looking at the coalitions in $S_{i, \bar{j}}$, either $\{i\}$ or $N \backslash\{j\}$ has the minimum profit. Similarly, looking at coalitions $S_{j, \bar{i}}$, either $\{j\}$ or $N \backslash\{i\}$ has the minimum profit. Formally, it is an exchange of minima and an application of Lemma 9, as follows

$$
\begin{gathered}
\min _{S \in S_{i, \bar{j}}} p\left(x^{\prime}, S\right)=\min _{S \in S_{i, \bar{j}}}\{\min \{x(S), x(N \backslash S)-c(N \backslash S)\}\}= \\
\min \left\{\min _{S \in S_{i, \bar{j}}}\{x(S)\}, \min _{S \in S_{i, \bar{j}}}\{x(N \backslash S)-c(N \backslash S)\}\right\}=\min \left\{x^{\prime}(i), c(j)-x^{\prime}(j)\right\}
\end{gathered}
$$

A similar calculation shows that

$$
\min _{S \in S_{j, \bar{i}}} p\left(x^{\prime}, S\right)=\min \left\{x^{\prime}(j), c(i)-x^{\prime}(i)\right\}
$$

To finish the proof, we have show that

$$
\min \left\{x^{\prime}(i), c(j)-x^{\prime}(j)\right\} \geq \min \left\{x^{\prime}(j), c(i)-x^{\prime}(i)\right\}
$$

These minima depend only on the payoff of $i$ and $j$, no other coalitions are involved. Since we have a disconnected hydraulic we may forget about the other vessels for the moment and concentrate on the vessel system of $\{i\} \cup\{j\}$. In this context (3) is equivalent 
to saying that $p\left(x^{\prime},\{i\}\right) \geq p\left(x^{\prime},\{j\}\right)$. We selected $x^{\prime}$ in such a way that $x^{\prime}(i)$ is not less than the CG-consistent share of $i$ and $x^{\prime}(j)$ is not more than the CG-consistent share of $j$. We have seen that the Talmud rule is consistent and for the restricted game this implies that $i$ gets at least as much as her CG-consistent share. By Lemma 11, the CG-consistent solution of the restricted game gives the same profit to both players, hence the profit of $i$ is not less in this game than that of $j$, and this is exactly what we had to prove.

To illustrate the power of the hydraulic approach, we repeat the somewhat tricky argument of the last paragraph in the language of the vessels. Consider disconnected hydraulic corresponding to $x$. As $i$ has more and $j$ has less than their respective CGconsistent shares, the water level according to $x$ is higher in the $i$ th vessel than in the $j$ th one.

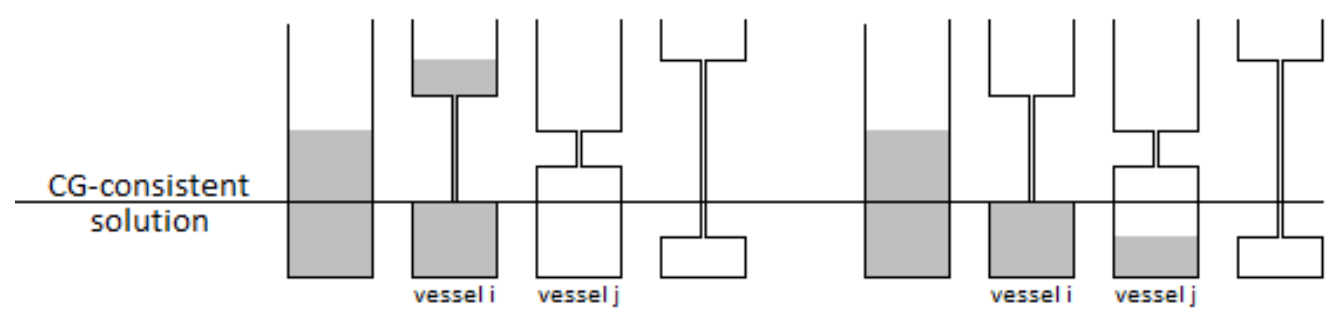

Figure 6: Before and after the water exchange

To obtain solution $x^{\prime}$ from $x$, we let some water from vessel $i$ into vessel $j$. We stop as soon as any of the levels reach the common water level of the CG-consistent solution. Now $x^{\prime}$ still has the property that the water is higher in vessel $i$ than in vessel $j$. Lemma 11 shows that equal water level means equal profits in two vessel hydraulics. Therefore the $x^{\prime}$-profit of $i$ is more than the $x^{\prime}$-profit of $j$ for the two vessel hydraulic, just as we claimed.

Remark: Though the above proof works without introducing the hydraulic framework, some statements, like observation 3 and 6 are more transparent this way. We think that the main advantage of hydraulics is that it makes easier to interpret notions like profit and nucleolus and helps to find and prove the right statements.

\section{References}

[1] Aumann, R., Game Theory in the Talmud, Research bulletin Series on Jewish Law and Economics (2003)

[2] Aumann, R., Maschler, M., Game theoretic analysis of a bankruptcy problem from the Talmud, Journal of Economic Theory 36 (1985) 195-213. 
[3] Benoît, J.-P., The nucleolus is contested-garment-consistent: a direct proof, Journal of Economic Theory 77 (1997) 192-196.

[4] Herrero, C., Villar, A., The three musketeers: four classical solutions to bankruptcy problems Mathematical Social Sciences 39 (2001) 307-328.

[5] Hokari, T., Thomson, W., On properties of division rules lifted by bilateral consistency Journal of Mathematical Economics, 44/11 (2008) 1057-1071.

[6] Kaminski, M., Hydraulic rationing, Mathematical Social Sciences 40 (2000) 131-155.

[7] Moreno-Ternero J. D., Villar A., The Talmud rule and the securement of agents' awards, Mathematical Social Sciences 47 (2004) 245-257.

[8] O'Neill, B., A problem of rights arbitration from the Talmud Mathematical Social Sciences 2 (1982) 345-371.

[9] Schmeidler, D., The Nucleolus of a Characteristic Function Game SIAM Journal on Applied Mathematics 17/6 (1969) 1163-1170

[10] Thomson, W., Axiomatic and game-theoretic analysis of bankruptcy and taxation problems: a survey, Mathematical Social Sciences 45 (2003) 249-297

[11] Thomson, W., Fair Allocation Rules, Chapter 21 of K. Arrow, A. Sen and K. Suzumura (eds.) The Handbook of Social Choice and Welfare, Vol. 2 (2011) North-Holland 